\title{
Translocated EspF protein from enteropathogenic Escherichia coli disrupts host intestinal barrier function
}

\author{
Barry P. McNamara, ${ }^{1}$ Athanasia Koutsouris, ${ }^{2}$ Colin B. O'Connell, ${ }^{3}$ \\ Jean-Philippe Nougayréde, ${ }^{1}$ Michael S. Donnenberg, ${ }^{1,3}$ and Gail Hecht ${ }^{2}$
}

${ }^{1}$ Division of Infectious Diseases, Department of Medicine, University of Maryland, Baltimore, Baltimore, Maryland, USA
${ }^{2}$ Section of Digestive and Liver Diseases, Department of Medicine, University of Illinois and
West Side Veterans Affairs Medical Center, Chicago, Illinois, USA
${ }^{3}$ Department of Microbiology and Immunology, University of Maryland, Baltimore, Baltimore, Maryland, USA

Address correspondence to: Michael S. Donnenberg, Division of Infectious Diseases, University of Maryland, Baltimore, 10 S. Pine Street, Baltimore, Maryland 21201, USA.

Phone: (410) 706-7560; Fax: (410) 706-8700; E-mail: mdonnenb@umaryland.edu.

Barry P. McNamara and Athanasia Koutsouris contributed equally to this work.

Michael S. Donnenberg and Gail Hecht contributed equally to this work.

Received for publication August 22, 2000, and accepted in revised form January 29, 2001.

\begin{abstract}
The mechanisms by which enteropathogenic Escherichia coli (EPEC), an important cause of diarrhea among infants in developing countries, induce symptoms are not defined. EPEC have a type III secretion system required for characteristic attaching and effacing changes that modify the cytoskeleton and apical surface of host cells. Infection of polarized intestinal epithelial cell monolayers by EPEC leads to a loss of transepithelial electrical resistance, which also requires the type III secretion system. We demonstrate here that EspF, a protein that is secreted by EPEC via the type III secretion system, is not required for quantitatively and qualitatively typical attaching and effacing lesion formation in intestinal epithelial cells. However, EspF is required in a dose-dependent fashion for the loss of transepithelial electrical resistance, for increased monolayer permeability, and for redistribution of the tight junction-associated protein occludin. Furthermore, the analysis of EPEC strains expressing EspF-adenylate cyclase fusion proteins indicates that EspF is translocated via the type III secretion system to the cytoplasm of host cells, a result confirmed by immunofluorescence microscopy. These studies suggest a novel role for $\mathrm{EspF}$ as an effector protein that disrupts intestinal barrier function without involvement in attaching and effacing lesion formation.
\end{abstract}

J. Clin. Invest. 107:621-629 (2001).

\section{Introduction}

Diarrhea remains one of the leading causes of mortality in children in developing countries. Enteropathogenic Escherichia coli (EPEC) strains may be the leading bacterial cause of diarrhea among newborns and young infants in such nations (1).

Much progress has been made in the past decade in unraveling the molecular basis for EPEC interactions with host cells. EPEC strains are defined by the ability to induce an effect known as attaching and effacing and by the lack of production of Shiga toxins. Attaching and effacing is a characteristic ultrastructural lesion in host cells that consists of loss of microvilli, the formation of cuplike pedestals composed of actin and other cytoskeletal elements, and the intimate attachment of bacteria to the surface of the cells at the sites of these alterations $(2,3)$. Attaching and effacing ability is encoded on a pathogenicity island known as the locus of enterocyte effacement (LEE) that is composed of 41 open reading frames (4).

Several categories of genes from the LEE have been described. The esc and sep genes encode a type III secretion system (5). The espA, esp $B$, and $\operatorname{esp} D$ genes encode proteins secreted by this system that may form a translocation apparatus for delivery of effector molecules into host cells (6). EspB may also be an effector protein that disrupts the cytoskeleton (7). The eae gene encodes an outer membrane adhesin known as intimin, and tir encodes the translocated intimin receptor (Tir) to which intimin binds after the type III system inserts Tir in the host membrane (8). The LEE also encodes EspF, a protein of unknown function that is also secreted via the type III system (9). Deletion of the espF locus has no effect on the ability of EPEC to induce the accumulation of actin in HEp-2 cells at sites of bacterial attachment, a phenotype that is considered to be equivalent to the attaching and effacing effect (10). The function of EspF is therefore unknown.

The mechanisms by which EPEC cause diarrhea have not been identified. Because EPEC can colonize and destroy microvilli along the entire bowel, it has been proposed that malabsorption may lead to an osmotic diarrhea (11). Indeed, fasting leads to improvement of symptoms in some infants (12). However, other infants continue to have diarrhea despite total parenteral nutrition (13), and in volunteers the diarrhea develops rapidly while fasting (14), making this mechanism implausible as the sole cause of symptoms. Recently, a 
rapid and transient change in short-circuit current has been noted in polarized intestinal epithelial cells infected with synchronized cultures of EPEC, which may reflect a secretory diarrhea due to net movement of ions and fluid across the intestinal epithelium (15). A change in voltage potential in patch-clamped Caco-2 cells infected with EPEC could represent a similar phenomenon (16). It was also shown recently that enterovirulent strains of E. coli, including EPEC, upregulate receptors for the neuropeptide galanin-1, which in turn stimulates chloride secretion (17). Finally, a number of laboratories have reported that infection of polarized intestinal epithelial cell monolayers with EPEC leads to a dramatic drop in transepithelial electrical resistance (TER) $(15,18-20)$. This drop in TER is accompanied by increased flux of paracellular fluid markers, altered distribution of the tight junction protein $\mathrm{ZO}-1$ and is not accompanied by death of the cells in the monolayer. It has therefore been proposed that EPEC diarrhea may be due, in part, to loss of intestinal barrier function with movement of ions and fluid from the intestinal submucosa to the lumen. Since EPEC induces all three of these effects, loss of microvilli, ion fluxes, and loss of tissue resistance, it has not been possible to determine the relative contributions of these mechanisms to the pathogenesis of diarrhea. The purpose of this study was to further define the role of $\mathrm{EspF}$ in EPEC pathogenesis by studying its effects on attaching and effacing and TER in a polarized intestinal epithelial cell monolayer and by determining whether it is translocated into epithelial cells.

\section{Methods}

Bacterial strains, plasmids and culture conditions. Wildtype EPEC strain E2348/69 (21), strain UMD874, which has a deletion that extends from the end of ORF29, just upstream of espF, to the end of the LEE that removes espF and the remnants of a transposon, and strain UMD876, which has a deletion that extends from the end of espF to the end of the LEE, have been described previously (9). Strain UMD872 has a disruption in the espA gene (22), strain UMD870 has a disruption of the espD gene (23), and strain CVD452 has a disruption in the escN gene (5). Plasmid pBPM32 was constructed by amplifying the espF gene using DeepVent polymerase (New England Biolabs Inc., Beverly, Massachusetts, USA) and primers Donne-335 (5'-GCG GGA TCC CCC TTT CTT CGA TTG CTC ATA G-3') and Donne-336 (5'-CAT GCC ATG GTT AAT GGA ATT AGT AAC-3'). The PCR product was cloned into pTEB68, a derivative of plasmid pTrc 99A (Amersham Pharmacia Biotech, Piscataway, New Jersey, USA) that was modified to allow the addition of a carboxyl-terminal hexahistidine tag. However, sequencing of $\mathrm{pBPM} 32$ revealed the presence of a frame-shift mutation that resulted in the inclusion of only three histidine codons as well as 13 additional codons to the $3^{\prime}$ end of espF. Primers Donne-355 (5'-CGG GAT CCA TAT AAA GAG GCA TAA ATT ATG-3') and Donne-356 (5'-AAC TGC AGC CCT TTC TTC GAT TGC TCA TAG-3') were used to amplify the espF gene for fusion to the amino-terminus of cya, which encodes the catalytic domain of Bordetella pertussis adenylate cyclase, in plasmid pBScya (24) to create pBPM37. Similarly, primer Donne-355 was used with primer Donne-338 (5'-AGG GCC CGG GAG TAA ATG AAG TCA CCT G-3') to create a fusion between the first 73 codons of espF and cya to create plasmid pBPM36. Recombinant plasmids were introduced into EPEC strains by electroporation as described (9).

Bacteria were routinely cultured in Luria-Bertani (LB) broth or on LB plates. Ampicillin was added at 200 $\mu \mathrm{g} / \mathrm{ml}$ and chloramphenicol at $25 \mu \mathrm{g} / \mathrm{ml}$ when needed to maintain plasmids.

Tissue culture. HeLa cells (CCL2; American Type Culture Collection, Rockville, Maryland, USA) were grown as described (25). T84 cells were grown in a 1:1 (vol/vol) mixture of Dulbecco-Vogt modified Eagle's medium and Ham's F-12 supplemented with 6\% newborn calf serum, then passaged, and plated on collagen-coated filters, as previously described (26).

Electron microscopy. Overnight bacterial cultures were diluted (1:33) in serum- and antibiotic-free T84 medium containing $0.5 \%$ mannose and grown to midlogarithmic growth phase. The bacterial suspension was pelleted and resuspended in culture medium. Bacteria (approximately $5 \times 10^{7} \mathrm{CFU}$ ) were added to the apical surface of T84 monolayers grown on collagen-coated permeable supports (Transwells; Corning-Costar Corp., Cambridge, Massachusetts, USA). Infected monolayers were incubated in antibiotic- and serum-free medium at $37^{\circ} \mathrm{C}$ in an atmosphere of $95 \%$ air, $5 \% \mathrm{CO}_{2}$ for 6 hours. The filter units were coded to blind the investigator to their identity and processed for electron microscopy as described previously (25). Attaching and effacing activity was quantified as described previously (27). Proportions were compared with the $\chi^{2}$ test.

Immunoblots. Immunoblots of bacterial pellets and supernatants for detection of EspF were performed and developed with enhanced chemiluminescence (ECL) detection reagents as described previously (9). Polyvinylidene fluoride membranes were cut horizontally. The lower half was probed as described with an affinity-purified anti-EspF antiserum, while the upper half was probed with mAb 5D1 against the catalytic domain of adenylate cyclase at a dilution of 1:2000 (28). For examination of EspF expression by the espF mutant containing pBPM32, isopropyl $\beta$-D-thiogalactopyranoside (IPTG) at various concentrations was added for 2 hours after the initial incubation. Preparations containing both bacterial pellet and supernatant proteins were obtained by addition of trichloroacetic acid directly to the culture. To quantify expression, blots were digitized and analyzed using Scion Image software (Scion Corp., Frederick, Maryland, USA).

Adenylate cyclase reporter assay. Overnight cultures of bacterial strains were diluted 1:100 in DMEM/F12 (Life Technologies Inc., Grand Island, New York, USA) and 
cultured with aeration at $37^{\circ} \mathrm{C}$ for 4 hours. Bacteria were diluted in DMEM/F12 to a concentration of approximately $2.5 \times 10^{7} \mathrm{CFU} / \mathrm{ml}$ and added at an moi of 100:1 to HeLa or T84 cells. The HeLa cells were pretreated for 30 minutes with cytochalasin D $(2 \mu \mathrm{g} / \mathrm{ml})$ to block bacterial invasion (29). Cells were incubated at $37^{\circ} \mathrm{C}$ for 1.5 hours in an atmosphere of $95 \%$ air, $5 \% \mathrm{CO}_{2}$. After infection, cells were washed twice with cold medium, and $400 \mu$ lof $\mathrm{HCl}(50 \mathrm{mM})$ was added to each well. Cells were removed with a rubber policeman, boiled for 5 minutes, and neutralized by the addition of $24 \mu \mathrm{l}$ $0.5 \mathrm{M} \mathrm{NaOH}$. A sample $(10 \mu \mathrm{l})$ of each lysate was retained for determination of protein concentration by the bicinchoninic acid method (Pierce Chemical Co., Rockford, Illinois, USA). Ethanol (800 $\mu \mathrm{l})$ was added to each sample for 5 minutes, and the samples were centrifuged (11,000 $\mathrm{g}$ for 5 minutes). The supernatants were dried in a SpeedVac (Savant Instruments Inc., Holbrook, New York, USA) for 2 hours and stored overnight at $-20^{\circ} \mathrm{C}$. The concentration of cAMP for each sample was determined using a commercial ELISAbased kit (BioTrak; Amersham Pharmacia Biotech) according to the directions stipulated in protocol one of the manufacturer's instructions. Reactions were terminated after 30 minutes by the addition of $1 \mathrm{M} \mathrm{H}_{2} \mathrm{SO}_{4}$. A linear standard curve was constructed using known concentrations of BSA for protein determinations, and a logarithmic standard curve was constructed using known concentrations of cAMP for the ELISA. A single value of cAMP concentration per milligram of protein was calculated for each sample. When necessary to yield values within the range of the standards, samples were diluted tenfold or 100 -fold in assay buffer before assay.

Confocal microscopy. HeLa cells were incubated on coverslips that were placed in 24-well plates in an atmosphere of $95 \%$ air, $5 \% \mathrm{CO}_{2}$ until $85 \%$ confluence was reached. Cells were pretreated with cytochalasin D and infected with EPEC at an moi of 100:1 as described for cAMP assays. After 1 hour, the cell monolayers were washed three times with PBS and incubated before fixation with wheat germ agglutinin (WGA) conjugated to Alexa $488(25 \mu \mathrm{g} / \mathrm{ml}$; Molecular Probes Inc., Eugene, Oregon, USA) in PBS for 5 minutes to label HeLa cell surface carbohydrates. Cells were then fixed with $3 \%$ formaldehyde and permeabilized with $0.5 \%$ Triton-X100 for 3 minutes. The fixed monolayers were stained with 4', 6'-diamidino-2-phenyl-indole (DAPI; Sigma Chemical Co., St. Louis, Missouri, USA) $(5 \mu \mathrm{g} / \mathrm{ml}$ in $\mathrm{H}_{2} \mathrm{O}$ ) for 1 hour at room temperature to label adherent bacteria and host cell nuclei. The monolayers were then blocked overnight at $4{ }^{\circ} \mathrm{C}$ in $1 \% \mathrm{BSA}$ and $0.2 \%$ sodium azide. An affinity-purified anti-EspF Ab (9) was used at a dilution of 1:200 in 1\% BSA in PBS and detected with an anti-rabbit $\operatorname{IgG} \mathrm{Ab}$ conjugated to lissamine rhodamine B (Molecular Probes Inc.) at a dilution of 1:200 in $1 \%$ BSA-PBS. The samples were examined with a Zeiss LSM410 confocal laser scanning microscope (Carl Zeiss Inc., Thornwood, New York, USA) and analyzed using LSM410 software as described previously (30).
Measurement of transepithelial electrical resistance and mannitol flux. T84 cells were grown to confluence on permeable, collagen-coated filters (Corning-Costar Corp.) and infected as described for electron microscopy. A simplified apparatus for measuring electrophysiological parameters described by Madara et al. (31) was used to measure TER. The cells were infected as described for electron microscopy with bacteria at moi from 10 to 1,000. After 1 hour, media were aspirated and monolayers were gently washed to remove all nonadherent bacteria as described previously (32). Fresh tissue culture medium was added and monolayers were incubated for the indicated times. TER was determined by passing $25 \mu \mathrm{A}$ of current, measuring the resulting voltage deflection, and applying Ohm's law $(V=I R)$ to calculate resistance. To measure changes in paracellular permeability, monolayers were infected as above. After 5 hours of infection, monolayers were placed in Ringer's solution containing $10 \mathrm{mM}$ glucose and $5 \mathrm{mM}$ cold mannitol and allowed to equilibrate for 30 minutes. Apical medium was removed and replaced with Ringer's solution as above, but containing $0.4 \mu \mathrm{Ci}$ of $\left[{ }^{3} \mathrm{H}\right]$-mannitol per filter. Incubation was continued for 1 hour. Basal medium was collected and counted in a scintillation counter. Mannitol flux is expressed as percentage of uninfected controls. To assess cell death, monolayers were monitored in parallel for release of lactate dehydrogenase (LDH) using a commercially available kit (Cytotoxicity Detection Kit; Roche Molecular Biochemicals, Mannheim, Germany).

Immunofluorescence. T84 monolayers were grown on glass coverslips and infected for 6 hours at an moi of 500 in the presence or absence of IPTG as described above. Occludin was visualized using a commercially available Ab (Zymed Laboratories Inc., South San Francisco, California, USA), as described (33).

\section{Results}

EspF is not required for attaching and effacing activity in an intestinal epithelial cell line. We reported previously that $\mathrm{EspF}$ is not required for EPEC to induce rearrangements of actin in infected human laryngeal carcinoma HEp-2 cells (9). To determine whether EspF is required for full attaching and effacing activity in differentiated intestinal epithelial cells, we infected polarized T84 intestinal epithelial cells with either wild-type EPEC strain E2348/69 or espF mutant strain UMD874 and determined the percentage of bacteria that were involved in attaching and effacing lesions by electron microscopy. Of a total of 192 wild-type bacteria observed in the samples, 95 (49\%) were engaged in attaching and effacing activity. Similarly, of a total of 122 espF mutant bacteria observed, 65 (53\%) were engaged in attaching and effacing activity $(P=0.59)$. Furthermore, we detected no qualitative difference in the nature of the attaching and effacing lesions induced by the wild-type and espF mutant strains (Figure 1).

EspF is translocated into epithelial cells. We reported previously that EspF is exported by EPEC via the type III secretion pathway (9). We used an adenylate cyclase 


\section{Figure 1}

Transmission electron micrographs of polarized T84 cells infected with (a) wild-type EPEC strain E2348/69 or (b) espF mutant strain UMD874. Typical attaching and effacing lesions are present in both micrographs (arrows). Bar, $1 \mu \mathrm{m}$.
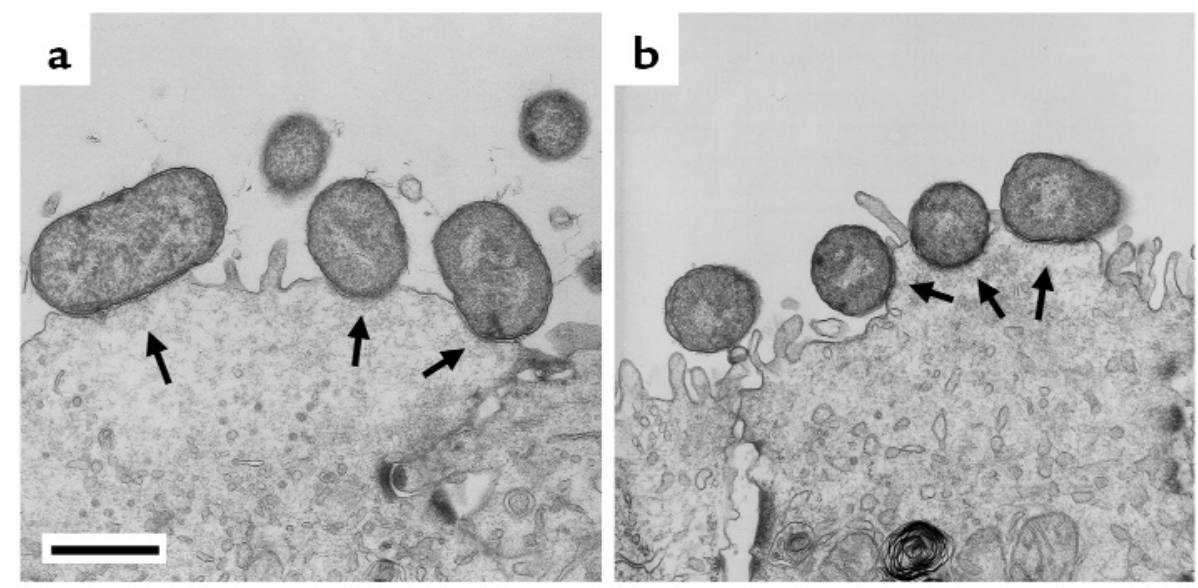

reporter system (34) to determine whether EspF is targeted to the host cell cytoplasm. Since the B. pertussis adenylate cyclase requires calmodulin for activity, fusion proteins that contain the catalytic portion of this enzyme have activity only if they are directed to the cytoplasm of host cells where calmodulin is abundant. We constructed a plasmid that has a fusion between fulllength espF and a portion of the cya gene encoding the catalytic domain of adenylate cyclase and introduced this plasmid into various EPEC strains. We also fused the first 73 codons of espF to cya, since in other systems the $5^{\prime}$ end of the gene is sufficient to allow effector molecules to be translocated via type III systems (35). We then infected HeLa cells with EPEC strains containing these constructs, prepared cell lysates, and assayed cAMP by ELISA. We found that cells infected with wildtype EPEC strain E2348/69 containing the full-length espF-cya fusion plasmid had levels of cAMP that were more than 100-fold greater than uninfected cells (Table 1). Similar levels were detected in cells infected with wild-type EPEC containing the plasmid encoding the truncated $\mathrm{EspF}^{\prime}$-adenylate cyclase fusion. In contrast, cells infected with mutant EPEC strains containing the full-length fusion plasmid, but with mutations in genes encoding components of either the type III secretion apparatus itself $(e s c N)$ or the translocation apparatus (espA), did not have elevated cAMP levels. Expression of wild-type EspF does not appear to be required for translocation of $\mathrm{EspF}$ as cells infected with an espF mutant expressing the EspF-adenylate cyclase fusion had high levels of cAMP. Similar results were obtained using T84 cells, indicating that EspF is also transported into a relevant intestinal epithelial cell line (Table 1) and in the presence or absence (data not shown) of cytochalasin, which blocks bacterial invasion by greater than $90 \%$ (29), indicating that the observed enzyme activity was not due to delivery from internalized bacteria. Western blots using Ab's directed either against EspF or against adenylate

\section{Table 1}

cyclase confirmed that the fusions were expressed by each strain and secreted into the supernatant by all strains except the type III secretion mutant, as expected (data not shown). Overall, these results indicate that the type III secretion system of EPEC delivers EspF to the host cell cytoplasm.

EspF can be detected within infected host cells by confocal microscopy. To confirm that EspF is translocated into host cells with an alternative method, we used confocal laser scanning microscopy to examine HeLa cells that had been pretreated with cytochalasin D and infected with various EPEC strains. For these studies we stained DNA with DAPI to visualize host cell nuclei and bacteria, EspF with specific antiserum, and host cell membranes with WGA conjugated to Alexa 488. Examination of multiple $0.5-\mu \mathrm{m}$ optical sections through individual cells clearly demonstrated EspF signal within host cells infected with wild-type EPEC strain E2348/69 (Figure 2). The signals were not homogeneous throughout the infected cells, but were instead present as focal accumulations within the cytoplasm, near but clearly separated from those of the infecting bacteria. In contrast to cells infected with wild-type EPEC, no signal was detected in cells infected with the espF mutant strain UMD874. Weaker EspF signals were detected in samples containing cells infected with $\operatorname{esc} N$ and $\operatorname{esp} A$ mutant strains, which have

Cyclic AMP levels in HeLa and T84 cells infected with EPEC strains expressing EspF-adenylate cyclase fusion proteins

\begin{tabular}{lcccc}
\hline Strain & Genotype & Plasmid & cAMP $\left(\text { pmol } \mathrm{mg}^{-1} \text { total protein }\right)^{\mathrm{A}}$ \\
& & & HeLa Cells & T84 Cells \\
Uninfected & - & - & $12.6 \pm 1$ & $10.1 \pm 1$ \\
E2348/69 & wild-type & pBPM36(EspF'-Cya) & $1580 \pm 82$ & $\mathrm{ND}$ \\
E2348/69 & wild-type & pBPM37(EspF-Cya) & $2460 \pm 180$ & $1857 \pm 200$ \\
UMD874 & espF & pBPM37(EspF-Cya) & $5260 \pm 250$ & $2547 \pm 177$ \\
UMD872 & espA & pBPM37(EspF-Cya) & $6.47 \pm 1$ & $9.63 \pm 1$ \\
CVD452 & escN & pBPM37 (EspF-Cya) & $6.50 \pm 4$ & $9.73 \pm 1$
\end{tabular}

AMean \pm SEM of triplicate samples from one experiment. Two additional experiments (for HeLa cells) or one additional experiment (for T84 cells) yielded similar results. EspF' indicates a truncated protein containing only the first 73 amino acids of EspF. ND, not done. 
mutations in components of the type III secretion system and the translocation apparatus, respectively. However, the signals in these samples were associated with the bacteria and the cell surface rather than within the host cells. Reconstruction of $y z$ and $x z$ planes through the depth of the epithelial cells confirmed that the EspF signal was within the cells infected with the wild-type strain only, while the signals were near the surface of cells infected with the escN and espA mutant strains (not shown). These studies confirm that the type III secretion and translocation system of EPEC delivers EspF to the interior of host cells.

EspF is required for EPEC-induced loss of transepithelial electrical resistance and increase in paracellular permeability in intestinal epithelial monolayers. Several investigators have reported that EPEC infection of polarized intestinal epithelial cells leads to a loss of TER (18-20). To deter- mine whether EspF plays a role in altering intestinal barrier function, we infected polarized T84 monolayers with EPEC strains at an moi of 500, removed nonadherent organisms as described, and monitored TER over time. This method of infection yields a final ratio of 50 cell-associated bacteria per cell at 6 hours (data not shown). As reported previously, we found a dramatic time-dependent loss of TER in monolayers infected with the wild-type EPEC strain E2348/69 (Figure $3 \mathrm{a}$ ). However, we detected no significant loss of TER in monolayers infected with espF mutant strain UMD874. The results with the espF mutant were similar to results obtained with the escN type III secretion mutant and the esp $A$ translocation mutant, which are incapable of delivering EspF to the host cell (data not shown). In contrast, mutant strain UMD876, which retains espF but has a deletion of the downstream trans-
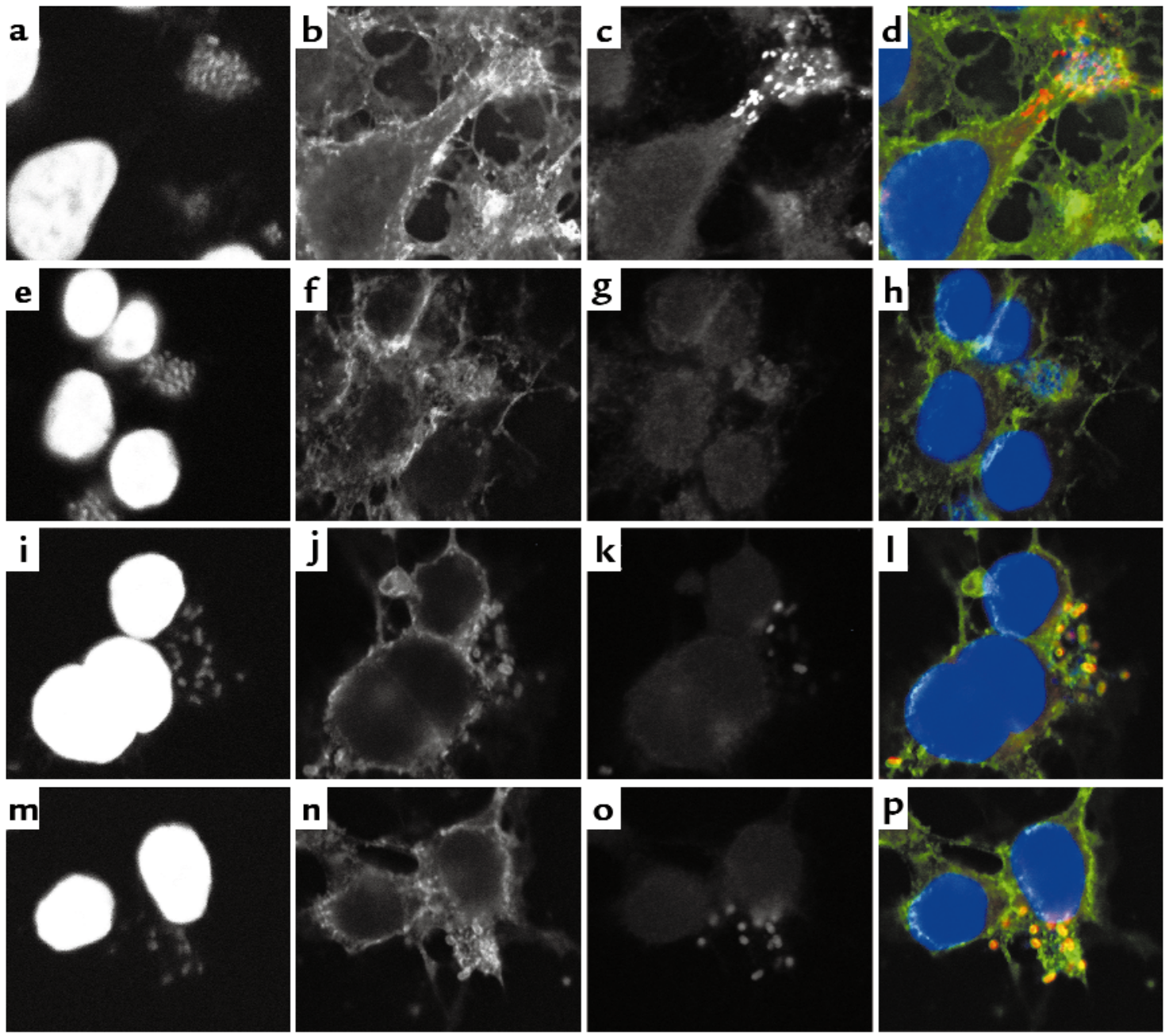

Figure 2

Confocal laser scanning microscopy of HeLa cells infected with wild-type and mutant EPEC strains. HeLa cells were infected in the presence of cytochalasin D with wild-type EPEC strain E2348/69 (a-d), espF mutant strain UMD874 (e-h), escN mutant strain CVD452 (i-I), and espA mutant strain UMD872 (m-p). Bacterial and cellular nucleic acid was labeled with DAPI (a, e, i, and $\mathbf{m})$, surface carbohydrates were labeled with WGA conjugated to Alexa $488(\mathbf{b}, \mathbf{f}, \mathbf{j}$, and $\mathbf{n})$, and EspF was labeled with affinity-purified EspF antiserum and detected with a secondary $\mathrm{Ab}$ against IgG conjugated to lissamine rhodamine $(\mathbf{c}, \mathbf{g}, \mathbf{k}$, and $\mathbf{o})$. Separate images of the same fields excited at different wavelengths as well as composite images $(\mathbf{d}, \mathbf{h}, \mathbf{I}$, and $\mathbf{p})$ are shown. In the composite images the bacteria and host cell nuclei appear blue, the cell surface carbohydrates appear green, and EspF appears red. 


\section{Figure 3}

The effect of EPEC strains on intestinal barrier function in T84 monolayers. (a) The TER of polarized T84 monolayers was measured using a simplified apparatus. Monolayers were left uninfected (open circles) or infected at an moi of 500 with wild-type EPEC strain E2348/69 (open squares); espF mutant strain UMD874 (open triangles); strain UMD876, which retains espF but contains a deletion of downstream sequences (filled circles); UMD874 containing plasmid pBPM19, which has the cloned espF gene (filled squares); or UMD874 with control plasmid PACYC184 (filled triangles). TER was measured over time and expressed as a percentage change from baseline values. Data shown represent the means ( \pm SEM) of four or five experiments containing triplicate or quadruplicate samples. The differences between monolayers infected with E2348/69 and UMD874 were significant at 2 hours, 4 hours, and 6 hours $(P=0.006,0.02$, and $<0.001$, respectively; Student's $t$ test). There was no significant difference between monolayers infected with E2348/69 and UMD876 or between those infected with E2348/69 and UMD874 (pBPM19). There was also no significant difference between uninfected monolayers and those infected with UMD874 or UMD874 (pACYC184). The mean \pm SEM TER of uninfected monolayers at baseline was $1138 \pm 67 \mathrm{ohms} \cdot \mathrm{cm}^{2}$. (b) TER was measured in monolayers infected for 6 hours at the indicated concentrations with wildtype EPEC strain E2348/69 (open squares) or with espF mutant strain UMD874 (open triangles) or left uninfected (open circle). Data shown represent the means ( \pm SEM) of three or four experiments containing triplicate samples. The differences between monolayers infected with E2348/69 and UMD874 were significant at moi of 250 $(P=0.04)$ and $500(P<0.001)$. (c) Mannitol flux was measured as described in Methods and expressed as a percentage of that recorded in uninfected monolayers. Monolayers were infected for 6 hours with wild-type EPEC strain E2348/69 (open squares) or espF mutant strain UMD874 (open triangles). Data shown represent the means $( \pm$ SEM) from two experiments containing triplicate samples. The differences between monolayers infected with E2348/69 and UMD874 were significant at moi of $500(P=0.02)$ and 1,000 $(P=0.001)$. a

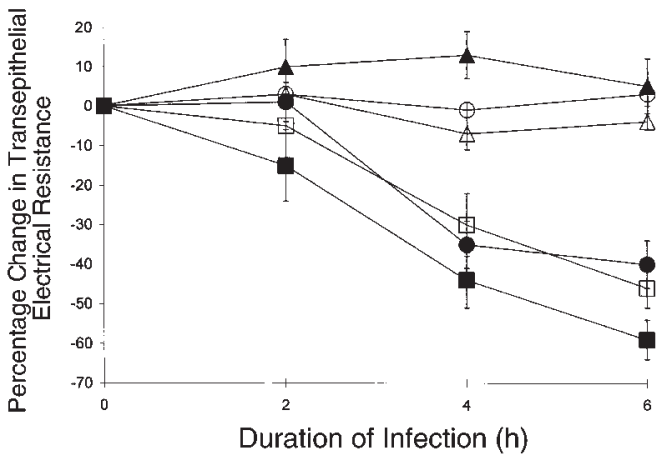

b

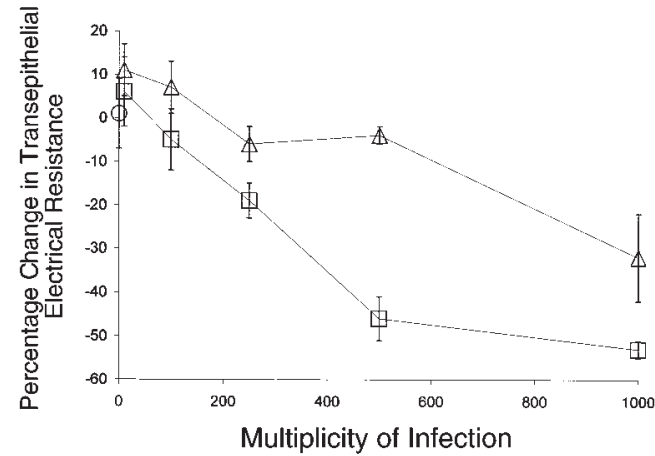

c

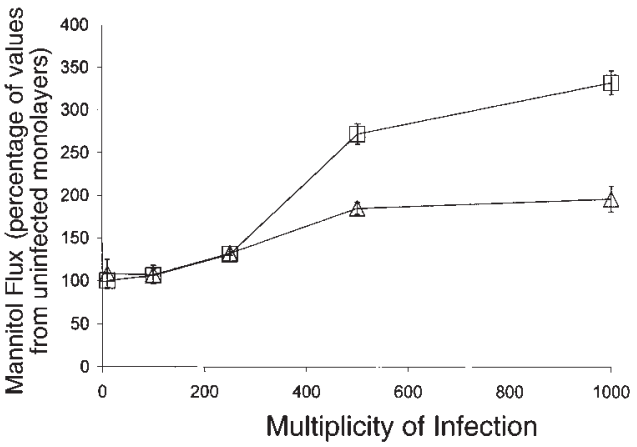

poson remnants, was able to induce a drop in TER. Furthermore, complementation of strain UMD874 with plasmid pBPM19 containing espF, but no downstream sequences (9), restored the ability of the mutant to induce a drop in TER. In contrast, introduction of pACYC184, the plasmid vector alone, had no effect. Complementation of the escN and espA mutants similarly restored the ability to induce a drop in TER (data not shown). To further evaluate the differences observed between the wild-type and espF mutant strain in ability to induce a drop in TER, we infected T84 cells for 6 hours with these strains at a range of moi. Whereas the wild-type strain induced a significant drop in TER at moi of 250 and greater, the espF mutant strain caused a significant drop in TER only at an moi of 1,000 (Figure 3b). Thus, we conclude that espF is critical for the EPEC-induced drop in TER.

TER is a highly sensitive method for measuring perturbations in monolayer permeability (36). To determine the physiological significance of the differences in TER observed between the wild-type and espF mutant strains, we measured the flux of mannitol, a compound that dif- fuses exclusively through the paracellular pathway, in T84 monolayers infected with these strains for 6 hours. We found a dose-dependent increase in mannitol flux that was much more pronounced in monolayers infected with the wild-type EPEC strain than in those infected with the esp $F$ mutant strain (Figure $3 c$ ). These differences were significant at moi of 500 and 1,000 (corresponding to concentrations of approximately 50 and 100 adherent organisms per cell, respectively, at the end of the incubation period). Thus, we conclude that espF is central to the EPEC-induced increase in paracellular permeability.

To determine whether the differences in TER and mannitol flux induced by the wild-type and espF mutant strains were due to differences in the ability of these strains to cause death of the cells in the monolayer, we measured LDH release at 6 hours. Approximately $2 \%$ of total LDH was released both by uninfected cells and by cells infected with the wild-type or the espF mutant at moi up to 500. At an moi of 1,000 the wild-type strain caused a release of $2.1 \pm 0.2 \%$ of total cellular LDH while the espF mutant strain caused a release of $2.4 \pm 0.1 \%$ of total cellular $\mathrm{LDH}(P=0.2)$. 
a
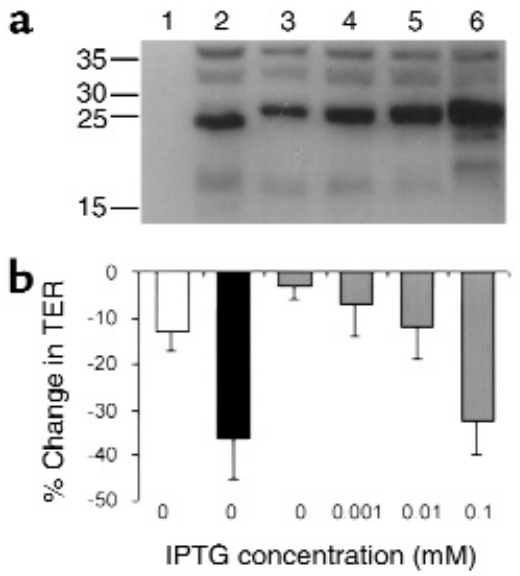

\section{Figure 4}

The level of expression of recombinant EspF correlates with ability of EPEC strains to induce a drop in TER in T84 monolayers. Bacteria were grown in the presence or absence of IPTG to induce EspF expression. Cultures were (a) concentrated and examined by immunoblot for EspF expression, or (b) used to infect T84 cells for 4 hours, and the change in TER was determined. Lane 1 in $\mathbf{a}$ and the open bar in $\mathbf{b}$ represent culture medium alone. Lane 2 and the filled bar represent wild-type EPEC strain E2348/69 in the absence of IPTG. Lanes 3-6 and the gray bars represent espF mutant strain UMD874 containing plasmid pBPM32 grown in the absence (lane 3 ) and the presence of varying concentrations of IPTG (lanes 4-6), as indicated. Data in b represent the mean $( \pm$ SEM) of two experiments with triplicate samples. The difference between monolayers infected with UMD874 (pBPM32) in the absence of IPTG and those infected with UMD874 (PBPM32) in the presence of $0.1 \mathrm{mM}$ IPTG was significant $(P=0.04)$.

Thus, the differences between these strains in their ability to disrupt intestinal barrier function are not due to differences in their ability to kill cells.

We noted in these experiments that the mutant strain complemented with the plasmid containing the espF gene consistently induced a greater loss of TER than did the wild-type strain. To determine whether there is a direct relationship between the levels of EspF expressed and the loss of TER, we transformed the espF mutant strain with plasmid PBPM32, which contains the espF gene under control of an inducible trc promot- er. We then infected monolayers with this strain in the presence of a range of concentrations of the inducer, IPTG. We found a direct correlation between the concentration of IPTG, the expression of EspF, and the drop in TER (Figure 4). In fact, the correlation coefficient for the relationship between the amount of recombinant EspF expressed (as measured in arbitrary units from the digitized blot image) and the percentage fall in TER was 0.955. Expression of native EspF from wild-type EPEC resulted in a greater loss in TER for the amount of protein expressed than did expression of the recombinant protein in the espF mutant, a difference that we attribute to the altered sequence of the recombinant protein (see Methods), which may have affected its activity. Thus, we conclude that the level of EspF expression is directly related to the drop in TER.

EspF is required for EPEC-induced disruption in the distribution of occludin in intestinal epithelial monolayers. To determine whether the observed electrophysiological effect of EspF on intestinal epithelial cell monolayers has a morphological counterpart, we examined the distribution of occludin, a transmembrane tight-junctions protein that contributes to barrier function, in cells infected with EPEC strains. As reported previously (33), the wild-type EPEC strain induces a change in occludin distribution from a uniform band outlining the cell junctions to a discontinuous beaded pattern outlining the cells with increased cytoplasmic staining (Figure 5). In contrast, cells infected with the espF mutant containing the inducible espF gene on pBPM32 in the absence of IPTG retained the uniform pattern of occludin staining. The addition of IPTG to induce expression of EspF from the plasmid caused a dosedependent relocalization of occludin such that a beaded pattern was apparent at a concentration of $0.01 \mathrm{mM}$ IPTG and a beaded and cytoplasmic pattern similar to that observed in cells infected with the wild-type strain at a concentration of $0.1 \mathrm{mM}$ IPTG.

\section{Discussion}

Since the initial identification of the gene that encodes it, EspF has been suggested to play a direct role in inter-

\section{Figure 5}

EspF is required for EPEC-induced changes in the distribution of occludin. T84 cells were left uninfected in the absence (a) or presence (b) of IPTG, infected with wild-type EPEC strain E2348/69 (c), or infected with the espF mutant strain UMD874 containing plasmid PBPM32 in the absence of IPTG (d) or in the presence of $0.01 \mathrm{mM}(\mathbf{e})$ or $0.1 \mathrm{mM}$ (f) IPTG. Occludin was visualized by immunofluorescence using a polyclonal Ab. Note the progressive redistribution of occludin corresponding to the IPTG concentration: a beaded pattern is seen with the lower concentration (e) and a shift from the region of the tight junction to the cytoplasm is seen with the higher concentration (f) similar to that observed with infection by wild-type EPEC (c).
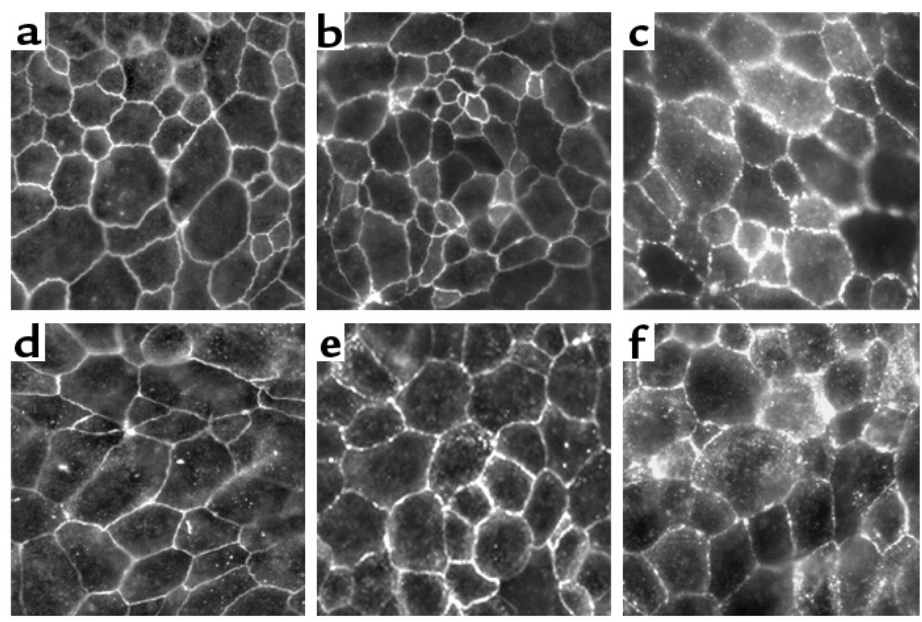
acting with host cells (37). The observations that EspF has three repeats of a proline-rich sequence that is similar to SH3-binding domains and that EspF is secreted by a type III apparatus have led to the hypothesis that EspF interacts inside cells directly with a host protein. However, the lack of an effect of deleting the espF gene on EPEC adhesion, attaching and effacing activity, and invasion cast doubt on the importance of EspF in pathogenesis (9). In this manuscript we describe a phenotype for the espF mutant and suggest a role for EspF in infection. By quantifying the ability of an espF mutant to form attaching and effacing lesions in polarized intestinal cell monolayers, we confirmed and extended previous findings that indicated that EspF is not required for attaching and effacing ability. However, despite its ability to induce morphologically normal attaching and effacing lesions, we found that the espF mutant is deficient in its ability to disrupt electrical resistance across a polarized monolayer of intestinal epithelial cells. This deficiency was associated with a reduced ability to enhance the transepithelial flux of mannitol, a marker for paracellular permeability, and with an inability to induce the redistribution of the tight junction associated protein occludin. In addition, we determined by two methods that EspF is translocated by EPEC into host cells, a result that would be expected of a protein secreted via the type III apparatus that functions as an effector molecule. Together, these results suggest a novel role of EspF in EPEC pathogenesis: an effector molecule required for disrupting intestinal epithelial barrier function.

Several laboratories have documented the ability of EPEC to cause a dramatic drop in TER $(15,18-20)$. Previous studies have described the loss of the ability to induce this drop in TER in mutants partially or totally deficient in adherence or attaching and effacing activity. In contrast, the current report represents the first identification of a mutant that is deficient in the ability to induce a fall in TER, but retains full adherence and attaching and effacing activity. The results of complementation studies using an inducible espF gene indicate that the amount of EspF produced is closely correlated with the degree by which TER falls. The observed changes in TER also correlated well with changes in occludin distribution. These relationships are consistent with a direct role of $\mathrm{EspF}$ in causing a defect in barrier function. Furthermore, we observed that mutants with defects in the type III secretion apparatus or the translocation apparatus are unable to deliver EspF to the host cell or disrupt tight junction permeability. Thus it is likely that the previously described inability of mutants with defects in adherence or attaching and effacing activity to induce a fall in TER is attributable to their inability to deliver sufficient EspF into host cells. The effect of the espF mutation on the ability of EPEC to induce a fall in TER appears to be relevant to other EPEC strains, because a similar mutation that we engineered in a recent clinical isolate of EPEC from Brazil had a similar effect (data not shown).
Previous studies have shown that the drop in tissue resistance and altered occludin distribution are reversible when antibiotics are applied to kill the adherent bacteria $(18,33)$. Furthermore, the drop in TER is not associated with an increase in LDH levels in the medium, indicating that the loss of tissue resistance is not due to death of the host cells (19). We confirmed these findings and, in addition, found no difference between the wild-type and esp $F$ mutant strains in their ability to damage cells in the monolayer.

While we demonstrated that EspF is necessary for disruption of barrier function by EPEC, we did not prove that $\mathrm{EspF}$ is solely responsible for this function. At the highest doses tested, even the esp $F$ mutant caused a drop in TER. This result may indicate a residual ability of the espF mutant to affect barrier function or may be a nonspecific effect of heavy bacterial infection. Attempts to determine whether EspF is sufficient to cause a drop in TER by transfecting monolayers with an EspF expression vector have yielded inconsistent results (data not shown). However, the dose-response relationships we observed between the level of EspF expression and both the drop in TER and altered localization of occludin are consistent with a direct effect.

For EspF to be directly involved in disrupting TER, the protein would have to interact with a specific surface receptor or be translocated into host cells. The results of the adenylate cyclase fusion assays indicate that at least at the carboxyl-terminal two-thirds of the protein is exposed to the cytoplasm of HeLa and T84 cells, since fusions containing only the first 73 amino acids of EspF as well as full-length fusions yield high enzyme activity. Thus, the information required for EspF translocation resides in the first 73 codons of the gene. Since EspF lacks hydrophobic segments that might serve as transmembrane domains, it is likely that the entire protein is translocated to the cytoplasm. The results of confocal microscopy also show EspF inside host cells. As was reported previously for Tir and EspB, the other EPEC-secreted proteins translocated into host cells, the distribution of EspF is not homogeneous, as would be expected of a soluble cytoplasmic protein (8, $30,38)$. Thus, EspF appears to be sequestered, perhaps by the host proteins with which it interacts. An alternative possibility, that EspF is present in cells within a vacuole, seems less likely in light of the results of the adenylate cyclase fusion assays and the apparent lack of a transmembrane segment.

Despite decades of study, the mechanisms by which EPEC cause diarrhea remain unclear. A variety of pathogenic processes, including malabsorption due to loss of microvilli, active fluid secretion, and loss of barrier function, have all been implicated as potential factors contributing to EPEC diarrhea. However, until now it has not been possible to assess the relative contributions of these mechanisms to disease since these properties of EPEC have been closely linked. Having demonstrated that $\mathrm{EspF}$ is required for disrupting barrier function, but not for attaching and effacing activity, it may now 
be possible to separate some of these effects and assess the relative contributions of these mechanisms to diarrhea. A study comparing the virulence of an espF mutant with a wild-type strain in volunteers should provide critical information on the pathogenesis of EPEC infection.

\section{Acknowledgments}

We thank Eric Hewlett for the gift of the mAb against adenylate cyclase (generated with support from Public Health Service award AI-18000), Kim Barrett for the T84 cells, and T. Eric Blank for plasmid pTEB68. These studies were supported by Public Health Service awards AI-32074 and AI-37606 (to M.S. Donnenberg) and DK50694 (to G. Hecht) from the NIH and by VA Merit Review and VA Research Enhancement Awards Program from the Department of Veterans Affairs (G. Hecht).

1. Nataro, J.P., and Kaper, J.B. 1998. Diarrheagenic Escherichia coli. Clin. Microbiol. Rev. 11:142-201.

2. Moon, H.W., Whipp, S.C., Argenzio, R.A., Levine, M.M., and Giannella, R.A. 1983. Attaching and effacing activities of rabbit and human enteropathogenic Escherichia coli in pig and rabbit intestines. Infect. Immun. 41:1340-1351.

3. Finlay, B.B., Rosenshine, I., Donnenberg, M.S., and Kaper, J.B. 1992. Cytoskeletal composition of attaching and effacing lesions associated with enteropathogenic Escherichia coli adherence to HeLa cells. Infect. Immun. 60:2541-2543.

4. Elliott, S.J., et al. 1998. The complete sequence of the locus of enterocyte effacement (LEE) of enteropathogenic E. coli E2348/69. Mol. Microbiol. 28:1-4.

5. Jarvis, K.G., et al. 1995. Enteropathogenic Escherichia coli contains a putative type III secretion system necessary for the export of proteins involved in attaching and effacing lesion formation. Proc. Natl. Acad. Sci. USA. 92:7996-8000.

6. Knutton, S., et al. 1998. A novel EspA-associated surface organelle of enteropathogenic Escherichia coli involved in protein translocation into epithelial cells. EMBO J. 17:2166-2176

7. Taylor, K.A., Luther, P.W., and Donnenberg, M.S. 1999. Expression of the EspB protein of enteropathogenic Escherichia coli within HeLa cells affects stress fibers and cellular morphology. Infect. Immun. 67:120-125.

8. Kenny, B., et al. 1997. Enteropathogenic E. coli (EPEC) transfers its receptor for intimate adherence into mammalian cells. Cell. 91:511-520.

9. McNamara, B.P., and Donnenberg, M.S. 1998. A novel proline-rich protein, EspF, is secreted from enteropathogenic Escherichia coli via the type III export pathway. FEMS Microbiol. Lett. 166:71-78.

10. Knutton, S., Baldwin, T., Williams, P.H., and McNeish, A.S. 1989. Actin accumulation at sites of bacterial adhesion to tissue culture cells: basis of a new diagnostic test for enteropathogenic and enterohemorrhagic Escherichia coli. Infect. Immun. 57:1290-1298.

11. Rothbaum, R.J., Partin, J.C., Saalfield, K., and McAdams, A.J. 1983. An ultrastructural study of enteropathogenic Escherichia coli infection in human infants. Ultrastruct. Pathol. 4:291-304.

12. Rothbaum, R., McAdams, A.J., Giannella, R., and Partin, J.C. 1982. A clinicopathological study of enterocyte-adherent Escherichia coli: a cause of protracted diarrhea in infants. Gastroenterology. 83:441-454.

13. Ulshen, M.H., and Rollo, J.L. 1980. Pathogenesis of Escherichia coli gastroenteritis in man: another mechanism. N. Engl. J. Med. 302:99-101.

14. Donnenberg, M.S., et al. 1993. The role of the eaeA gene in experimental enteropathogenic Escherichia coli infection. J. Clin. Invest. 92:1412-1417.

15. Collington, G.K., Booth, I.W., and Knutton, S. 1998. Rapid modulation of electrolyte transport in Caco-2 cell monolayers by enteropathogenic Escherichia coli (EPEC) infection. Gut. 42:200-207.

16. Stein, M.A., Mathers, D.A., Yan, H., Baimbridge, K.G., and Finlay, B.B. 1996. Enteropathogenic Escherichia coli (EPEC) markedly decreases the resting membrane potential of Caco- 2 and HeLa human epithelial cells. Infect. Immun. 64:4820-4825.

17. Hecht, G., et al. 1999. Pathogenic Escherichia coli increase Cl- secretion from intestinal epithelia by upregulating galanin-1 receptor expression.
J. Clin. Invest. 104:253-262.

18. Canil, C., et al. 1993. Enteropathogenic Escherichia coli decreases the transepithelial electrical resistance of polarized epithelial monolayers. Infect. Immun. 61:2755-2762.

19. Spitz, J., et al. 1995. Enteropathogenic Escherichia coli adherence to intestinal epithelial monolayers diminishes barrier function. Am. J. Physiol. Gastrointest. Liver Physiol. 268:G374-G379.

20. Philpott, D.J., McKay, D.M., Sherman, P.M., and Perdue, M.H. 1996. Infection of T84 cells with enteropathogenic Escherichia coli alters barrier and transport functions. Am. J. Physiol. Gastrointest. Liver Physiol. 270:G634-G645.

21. Levine, M.M., et al. 1978. Escherichia coli strains that cause diarrhoea but do not produce heat-labile or heat-stable enterotoxins and are non-invasive. Lancet. 1:1119-1122.

22. Kenny, B., Lai, L.-C., Finlay, B.B., and Donnenberg, M.S. 1996. EspA, a protein secreted by enteropathogenic Escherichia coli (EPEC), is required to induce signals in epithelial cells. Mol. Microbiol. 20:313-323.

23. Lai, L.C., Wainwright, L.A., Stone, K.D., and Donnenberg, M.S. 1997. A third secreted protein that is encoded by the enteropathogenic Escherichia coli pathogenicity island is required for transduction of signals and for attaching and effacing activities in host cells. Infect. Immun. 65:2211-2217.

24. Håkansson, S., et al. 1996. The YopB protein of Yersinia pseudotuberculosis is essential for the translocation of Yop effector proteins across the target cell plasma membrane and displays a contact-dependent membrane disrupting activity. EMBO J. 15:5812-5823.

25. Donnenberg, M.S., Yu, J., and Kaper, J.B. 1993. A second chromosomal gene necessary for intimate attachment of enteropathogenic Escherichia coli to epithelial cells. J. Bacteriol. 175:4670-4680.

26. Madara, J.L., Stafford, J., Dharmsathaphorn, K., and Carlson, S. 1987. Structural analysis of a human intestinal epithelial cell line. Gastroenterol. 92:1133-1145.

27. Anantha, R.P., Stone, K.D., and Donnenberg, M.S. 1998. The role of $\mathrm{BfpF}$, a member of the PilT family of putative nucleotide-binding proteins, in type IV pilus biogenesis and in interactions between enteropathogenic Escherichia coli and host cells. Infect. Immun. 66:122-131.

28. Lee, S.J., Gray, M.C., Guo, L., Sebo, P., and Hewlett, E.L. 1999. Epitope mapping of monoclonal antibodies against Bordetella pertussis adenylate cyclase toxin. Infect. Immun. 67:2090-2095.

29. Donnenberg, M.S., Donohue-Rolfe, A., and Keusch, G.T. 1990. A comparison of HEp-2 cell invasion by enteropathogenic and enteroinvasive Escherichia coli. FEMS Microbiol. Lett. 57:83-86.

30. Taylor, K.A., O'Connell, C.O., Luther, P.W., and Donnenberg, M.S. 1998. The EspB protein of enteropathogenic Escherichia coli is targeted to the cytoplasm of infected HeLa cells. Infect. Immun. 66:5501-5507.

31. Madara, J.L., Colgan, S.P., Nusrat, A., Delp, C., and Parkos, C.A. 1992. A simple approach to measurement of electrical parameters of cultured epithelial monolayers: use in assessing neutrophil epithelial interactions. Journal of Tissue Culture Methods. 14:209-216.

32. Savkovic, S.D., Koutsouris, A., and Hecht, G. 1996. Attachment of a noninvasive enteric pathogen, enteropathogenic Escherichia coli, to cultured human intestinal epithelial monolayers induces transmigration of neutrophils. Infect. Immun. 64:4480-4487.

33. Simonovic, I., Rosenberg, J., Koutsouris, A., and Hecht, G. 2000. Enteropathogenic Escherichia coli dephosphorylates and dissociates occludin from intestinal epithelial tight junctions. Cellular Microbiology. 2:305-315.

34. Sory, M.-P., and Cornelis, G.R. 1994. Translocation of a hybrid YopEadenylate cyclase from Yersinia enterocolitica into HeLa cells. Mol. Microbiol. 14:583-594.

35. Sory, M.P., Boland, A., Lambermont, I., and Cornelis, G.R. 1995. Identification of the YopE and YopH domains required for secretion and internalization into the cytosol of macrophages, using the cyaA gene fusion approach. Proc. Natl. Acad. Sci. USA. 92:11998-12002.

36. Madara, J.L., and Hecht, G.A. 1989. Tight (occluding) junctions in cultured (and native) epithelial cells. In Modern cell biology: functional epithelial cells in culture. K. Matlin, editor. Alan R. Liss Inc. New York, New York, USA. 131-169.

37. Donnenberg, M.S., Lai, L.C., and Taylor, K.A. 1997. The locus of enterocyte effacement pathogenicity island of enteropathogenic Escherichia coli encodes secretion functions and remnants of transposons at its extreme right end. Gene. 184:107-114.

38. Wolff, C., Nisan, I., Hanski, E., Frankel, G., and Rosenshine, I. 1998. Protein translocation into host epithelial cells by infecting enteropathogenic Escherichia coli. Mol. Microbiol. 28:143-155. 